\title{
Toxoplasmosis initially presenting as neurological sequelae of chimeric antigen receptor $T$-cell therapy
}

A 46-year-old male with relapsed B-cell acute lymphoblastic leukaemia following an allogeneic sibling haematopoietic stem cell transplant presented with neurological signs including limb weakness, imbalance, cognitive impairment and seizures. His most recent treatment had been with CD19 CAR-T cell therapy 4-months previously $\left(3 \times 10^{6} / \mathrm{kg}\right.$; he had previously received a first dose of 1 × $10^{6} / \mathrm{kg} 5$ months prior to the second dose). His underlying leukaemia remained at a level of low minimal residual disease by molecular monitoring. The differential diagnoses included CNS relapse of B-ALL, neurotoxicity secondary to CAR T-cell therapy and atypical infections.

A CT head scan showed an extensive white matter abnormality involving both frontal lobes and the corpus callosum. An MRI brain showed a large peripherally enhancing lesion within the right frontal lobe extending across the anterior corpus callosum and into the left frontal lobe (Figure 1).

The most likely cause was felt to be a malignant process, but in view of the complex history a brain biopsy was performed. H\&E staining showed a dense infiltrate of lymphocytes (mostly CD3 positive; CD20 negative); the CD68 stain confirmed macrophage infiltration. Immunostaining showed scattered Toxoplasma gondii tachyzoites. CSF was positive for Toxoplasma by PCR. The patient was commenced on Pyrimethamine (75mg once daily for 6 weeks, followed by $25 \mathrm{mg}$ once daily maintenance), Sulfadiazine (1.5g qds for 6 weeks followed by 500mg qds maintenance) and folinic acid (10mg once daily), and one year later he has made an excellent and almost complete neurological recovery with residual right arm proximal muscle weakness. On repeat imaging, the right frontal lesion has improved whereas the corpus callosum lesion remains stable.

Neurotoxicity following CD19 CAR-T cell therapy has been well described and usually presents with an encephalopathy syndrome, occasionally complicated by seizures. In this case, however, the cause of the neurological dysfunction was underlying cerebral toxoplasmosis. At the time of the transplant, the patient was Toxoplasma ( $\mathrm{IgG}$ ) seropositive whereas his donor was seronegative and so he had been receiving Azithromycin as prophylaxis.

This case emphasises the importance of performing a biopsy to obtain a histological diagnosis, particularly in those patients with profound immunosuppression who are receiving novel immunotherapies.

\footnotetext{
Maria A. V. Marzolini ${ }^{1}$, Zane Jaunmuktane ${ }^{2}$, Claire Roddie ${ }^{1}$, Maeve O'Reilly ${ }^{1}$, Peter Chiodini ${ }^{3}$ and Karl S. Peggs ${ }^{1}$

${ }^{1}$ Department of Haematology, UCL Cancer Institute and UCLH NHS Foundation Trust, London, UK. ${ }^{2}$ Division of Neuropathology, Institute of Neurology, The National Hospital for Neurology and Neurosurgery, Queen Square, London, UK. ${ }^{3}$ Department of Clinical Parasitology, UCLH NHS Foundation Trust, London, UK. Email: m.marzolini@ucl.ac.uk
} 
All authors contributed to the preparation of this manuscript. There are no conflicts of interest.

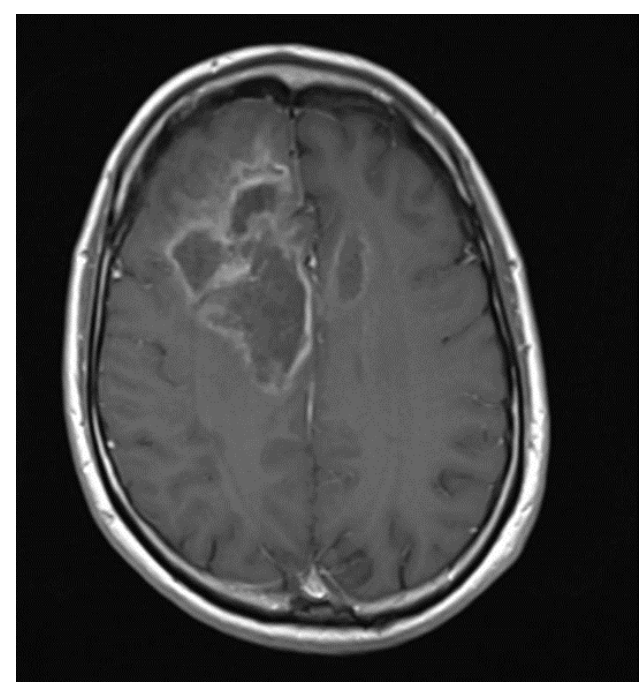

Figure 1: The MRI head scan (Transverse view) which showed the large, peripherally enhancing lesion within the right frontal lobe, extending across the corpus callosum and into the left frontal lobe. 